\title{
Neural Correlates of Correct and Errant Attentional Selection Revealed Through N2pc and Frontal Eye Field Activity
}

\author{
Richard P. Heitz, Jeremiah Y. Cohen, Geoffrey F. Woodman, and Jeffrey D. Schall \\ Department of Psychology, Vanderbilt Vision Research Center, Center for Integrative and Cognitive Neuroscience, Vanderbilt Brain \\ Institute, Vanderbilt University, Nashville, Tennessee
}

Submitted 7 July 2010; accepted in final form 27 August 2010

\begin{abstract}
Heitz RP, Cohen JY, Woodman GF, Schall JD. Neural correlates of correct and errant attentional selection revealed through N2pc and frontal eye field activity. J Neurophysiol 104: 2433-2441, 2010. First published September 1, 2010; doi:10.1152/jn.00604.2010. The goal of this study was to obtain a better understanding of the physiological basis of errors of visual search. Previous research has shown that search errors occur when visual neurons in the frontal eye field (FEF) treat distractors as if they were targets. We replicated this finding during an inefficient form search and extended it by measuring simultaneously a macaque homologue of an event-related potential indexing the allocation of covert attention known as the m-N2pc. Based on recent work, we expected errors of selection in FEF to propagate to areas of extrastriate cortex responsible for allocating attention and implicated in the generation of the m-N2pc. Consistent with this prediction, we discovered that when FEF neurons selected a distractor instead of the search target, the m-N2pc shifted in the same, incorrect direction prior to the erroneous saccade. This suggests that such errors are due to a systematic misorienting of attention from the initial stages of visual processing. Our analyses also revealed distinct neural correlates of false alarms and guesses. These results demonstrate that errant gaze shifts during visual search arise from errant attentional processing.
\end{abstract}

\section{IN T R O D U C T I O N}

Comprehensive models of cognition must account for patterns of both correct and errant behavior. This has proven difficult because errors can arise in many ways, both through faulty sensory processing and through hasty response preparation. Still, behavioral measures of errant responding have been of interest for many years (Rabbitt 1966), and leading models of perceptual decision making aim to account for errant behavior along with correct responding (Ratcliff and Rouder 1998). Unfortunately, these efforts are limited by a paucity of evidence identifying the neural correlates of errors. Understanding the neural activity associated with errant performance provides a more complete picture of the neurophysiological basis of behavior and offers important constraints on cognitive models (e.g., Purcell et al. 2010).

In this study, we obtained new information about how errors of visual search occur by recording the macaque homologue of the human N2pc using electrodes embedded in the macaque skull (Woodman et al. 2007) simultaneously with single units and local field potential (LFP) in macaque frontal eye field (FEF), an area recognized as contributing to attentional selection on the one hand (Cohen et al. 2009a; Sato and Schall 2003; Schall et al. 1995a) and saccade production on the other (Bruce

Address for reprint requests and other correspondence: J. D. Schall, Dept. of Psychology, Vanderbilt University, PMB 407817, 2301 Vanderbilt Pl., Nashville, TN 37240 (E-mail: jeffrey.d.schall@vanderbilt.edu). and Goldberg 1985; Hanes and Schall 1996; Ray et al. 2004; Schall 1991).

Previous research has demonstrated that the N2pc is a signature of covert attentional selection (Luck and Hillyard 1994; Luck et al. 1993) and can be used to monitor dynamic shifts of attention (Woodman and Luck 1999). The N2pc was discovered when human subjects performed visual search for a lateralized target stimulus (Luck et al. 1993). In humans, the $\mathrm{N} 2 \mathrm{pc}$ is observed as a greater negativity at electrode sites contralateral to the target stimulus, 175-200 ms after array onset. Importantly, the N2pc appears in tasks designed to prevent eye movements, occurs well before any manual responses are generated, and is even elicited by attended objects that do not require a response of any kind (Woodman and Luck 1999). Furthermore, because the N2pc is a lateralized component, a prerequisite for its emergence is a consistency in the direction of attentional orienting. If attention was directed in a haphazard fashion, no N2pc could be found because of the averaging across locations. Similarly, the N2pc does not emerge when conditions preclude attentional selection, such as when sensory input is data limited (Woodman and Luck 2003). Hence the N2pc reflects consistent, task related movements of covert attention apart from any overt response. We recently discovered that macaque monkeys exhibit a homologue of the human $\mathrm{N} 2 \mathrm{pc}$ (referred to henceforth as the $\mathrm{m}-\mathrm{N} 2 \mathrm{pc}$ ) that demonstrates identical functional characteristics (Woodman et al. 2007).

This study is the first to examine whether the m-N2pc occurs during visual search errors although FEF activity during such behavior has been well documented (Shen and Paré 2007; Thompson et al. 2005; Trageser et al. 2008). Briefly, FEF neurons incorrectly select distractor items prior to an errant saccade into the receptive field (RF). The equating of target selection in FEF with attention allocation (Armstrong et al. 2009; Kodaka et al. 1997; Sato and Schall 2003) requires that other indices of covert attention, such as the N2pc, mirror the neural activity known to occur in FEF during visual search. While we have recently demonstrated this for correct trials (Cohen et al. 2009a), it remains to be seen whether the relationship will continue to hold when processing breaks down. The placement of FEF as an important contributor to attentional orienting suggests a similar FEF neuron-m-N2pc mirroring on error trials. A demonstration that the m-N2pc tracks errant attentional orienting concordant with errant target selection in FEF would strengthen the hypothesis that FEF processing is closely related to the $\mathrm{m}-\mathrm{N} 2 \mathrm{pc}$ and thereby plays a role in covert orienting. 


\section{METHODS}

\section{Behavioral tasks and recording}

Two male macaques (Macaca radiata) were trained to perform visual search for a form-defined target among similar distractors (Cohen et al. 2009b). The monkeys were also trained to perform a memory-guided saccade task for use in characterizing the response properties of FEF single units (Bruce and Goldberg 1985; Hikosaka and Wurtz 1983). During the memory-guided task, monkeys began a trial by fixating for 750-1,000 ms. Then a circular target was presented for $100 \mathrm{~ms}$ at one of eight iso-eccentric locations. Eccentricity was adjusted based on the response properties of the neuron being recorded and target size was scaled with the cortical magnification factor. Following a delay of 500-1,000 ms, monkeys made a saccade to the remembered location of the now-absent target. Monkeys were rewarded for fixating the remembered location for $1,000 \mathrm{~ms}$ and were not rewarded when saccades were either not produced or made to an incorrect location. In the visual search task, monkeys were shown an iso-eccentric, circular array of rotated $\mathrm{T}$ and $\mathrm{L}$ shapes (see Fig. 1A). On a given session, either a $\mathrm{T}$ or $\mathrm{L}$ of a specific orientation was defined as the target. Distractor items were drawn from the nontarget set. The number of distractors presented on each trial was one, three, or seven (leading to set sizes of 2,4, and 8) and was determined randomly. Each trial began with monkeys fixating a central point for 750-1,000 ms. To earn liquid reward, monkeys had to make one

A
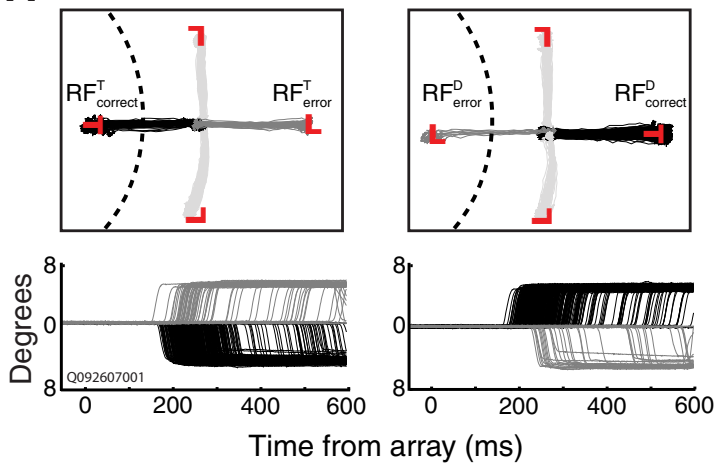

B
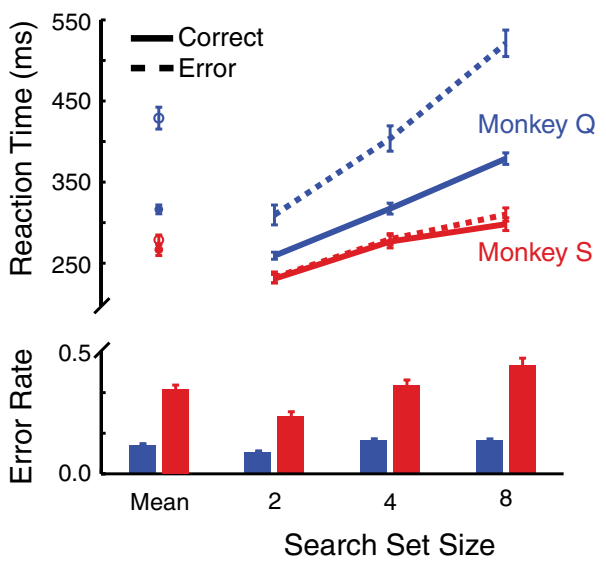

FIG. 1. A: saccade trajectories during visual search. Monkeys made saccades to a target $\mathrm{T}$ shape in 4-element array. For display, target and distractor items are colored red. The receptive field (RF) for all signals in this session was directly to the left of the fixation point, indicated by the dashed semicircle, not to scale. Below each plot are the $x$-dimension eye traces recorded from the eye coil signal, converted to degrees visual angle. Gray traces are errors $180^{\circ}$ from the target, black traces are correct trials to the target. $B$ : mean RT and error rate during the visual search task. Vertical bars represent SE. saccade directly to the target location and hold that position for $1,000 \mathrm{~ms}$.

Direction errors were those trials in which the monkey made a valid eye movement to a screen location containing a distractor when a target had been present. Target-present errors of other types (e.g., lack of any eye movement within 2,000 ms of target appearance, failure to maintain fixation in the target window for $1,000 \mathrm{~ms}$, eye movements to empty screen locations) were rare and were not analyzed further. Some sessions included catch trials where all display elements were distractor items. The proportion of catch trials was between 10 and $30 \%$ on these days. To earn a reward, monkeys had to maintain central fixation for $750 \mathrm{~ms}$. Thus the second type of error was a catch error in which an eye movement of any type was made. In some sessions, the catch trial display immediately disappeared at $750 \mathrm{~ms}$ when reward was delivered, whereas in other sessions, the displays remained visible for an additional $500 \mathrm{~ms}$ to eliminate any possibility for contamination due to an offset response. This had no effect on behavioral or neural data and patterns were identical across the two schemes.

Our analysis of FEF activity and the m-N2pc contrasted activity on correct trials with direction errors in which gaze shifted opposite to the actual target location (Fig. 1A). This selection of error trajectories allowed us to use the neuron-antineuron approach to quantify the target selection process (Britten et al. 1992; Thompson et al. 1996).

During aseptic surgery, monkeys were implanted with a head post, recording chambers, electroencephalogram (EEG) electrodes, and a subconjunctival eye coil under isoflurane anesthesia. Antibiotics and analgesics were administered postoperatively. All surgical and experimental procedures were in accordance with the National Institutes of Health Guide for the Care and Use of Laboratory Animals and approved by the Vanderbilt Institutional Animal Care and Use Committee.

Neurons and LFPs were recorded from FEF located in the rostral bank of the arcuate sulcus. FEF was identified by evoking saccades with $<50 \mu \mathrm{A}$ of microstimulation current. Neurons and LFPs were recorded simultaneously from both hemispheres using tungsten microelectrodes (2-4 M $\Omega, F H C$ ) and were referenced to a guide tube in contact with the dura. The EEG and the averaged event-related potentials (ERPs) were recorded from the monkeys using an array of electrodes implanted in the exterior mantle of the skull (Woodman et al. 2007). Spikes were sampled at $40 \mathrm{kHz}$; LFP and electroencephalographic (EEG) signals were sampled at $1 \mathrm{kHz}$. LFP and EEG signals were acquired in one of two ways. About half of the data for monkey $Q$ was band-pass filtered between 0.7 and $170 \mathrm{~Hz}$, amplified using a Plexon HST/8o50-G20 head-stage. The other half of monkey $Q$ 's data and nearly all of monkey S's data were band-pass filtered between 0.2 and $300 \mathrm{~Hz}$, amplified using a Plexon high-impedance HST/8o50-G1 head-stage. The differences in head-stage may account for the slight difference in visually evoked LFP polarization between the monkeys as low impedance head-stages are known to create distortions in LFPs (Nelson et al. 2008).

For each isolated neuron, we mapped the RF using activity during the memory-guided task. Cells were classified as visual or visuomovement (pure movement cells were not analyzed). Visual neurons had above-baseline activity in the $100 \mathrm{~ms}$ following stimulus onset but no modulation before a saccade while visuomovement neurons exhibited both a visual response and buildup activity before a saccade. LFP and EEG signals were assigned an RF based on hemisphere. That is, signals recorded in the right hemisphere were assigned left hemifield RFs, and vice versa. LFPs and EEGs were included only if they exhibited statistically significant selectivity across hemifields. Using a more restricted RF for the LFP and EEG, based on the concurrently recorded neuron's RF, did not change the data or conclusions qualitatively. We assessed selectivity by comparing activity on correct trials when a target fell in the $\mathrm{RF}\left(\mathrm{RF}_{\text {correct }}^{\mathrm{T}}\right)$ to that when a distractor appeared there $\left(\mathrm{RF}_{\text {correct }}^{\mathrm{D}}\right)$. Running Wilcoxon rank-sum tests were computed ms by ms, and a signal was categorized as selective if it 
reached significance for 10 consecutive $\mathrm{ms}$ at the 0.05 level. We verified this by computing selectivity using receiver-operating curves (ROC). Each point on an ROC curve reflects the probability that $\mathrm{RF}_{\text {correct }}^{\mathrm{T}}$ activity is greater than some criterion as a function of the probability that $\mathrm{RF}_{\text {correct }}^{\mathrm{D}}$ activity is greater than that criterion. In the case of single units, the criterion was incremented from 0 spike/s to the maximum firing rate observed across all trials in steps of 1 spike/s. For LFP and EEG signals, the criterion incremented from the smallest voltage observed to the largest voltage observed in steps of $10 \mu \mathrm{V}$. The area under this curve, at a given time point, can be interpreted as the ability of an ideal observer to determine whether the target was in the RF given only the neural data. If the ROC area reached 0.70 , the signal considered selective. Importantly, the neurons identified as being selective using running Wilcoxon tests were the same as those identified using ROC analyses. This ensured that the neural signals we analyzed were significantly tuned for target identity. We imposed this constraint only on correct trials, as error trials had significantly lower signal-to-noise ratio. This did not present a problem for our analyses as we were primarily interested in the direction of selectivity. While this undoubtedly included neurons with weak activity during error trials, doing so only made it more difficult for us to reject the null hypothesis.

ERP analyses were conducted with electrode pairs OL/OR and T5/T6 in the macaque analog of the human 10/20 system (Jasper 1958; Woodman et al. 2007) for monkeys $S$ and $Q$, respectively. A frontal electrode at position Fz was used as the EEG reference for both monkeys. We present only data from electrode OR and T6 in monkeys $S$ and $Q$ as skull electrode OL in monkey $S$ demonstrated a curiously inverted P1/N1 polarity change after a frontal craniotomy and was unreliable. Importantly, all patterns of $\mathrm{m}-\mathrm{N} 2 \mathrm{pc}$ data were verified using the left hemisphere T5 electrode of monkey $Q$; the left hemisphere data were identical to those from the right hemisphere.

\section{Data processing}

Spikes were convolved with a kernel resembling a postsynaptic potential (Thompson et al. 1996). LFP and ERP data were processed as follows. Trials on which signals saturated the amplifier were excluded from the analyses. Signals were baseline corrected using the average voltage in a $100 \mathrm{~ms}$ window ending at the time of array presentation. EEG signals were truncated trial-by-trial $20 \mathrm{~ms}$ before the saccade to eliminate any influence of the saccade artifact. This tended to increase noise levels; therefore ERP waveforms were filtered ( $50 \mathrm{~Hz}$ low-pass, zero-phase shift filter) for display purposes only. All statistics were computed on unfiltered data.

\section{RES ULTS}

We identified neuron, LFP, and ERP recordings that met the criteria described in the preceding text. Monkey $Q$ contributed 42 neurons, 31 LFPs, and 14 ERPs. Monkey $S$ contributed 19 neurons, 46 LFPs, and 19 ERPs.

\section{Behavior}

Figure $1 A$ depicts a typical visual search session in which a $\mathrm{T}$ (rotated $90^{\circ}$ ) was the target stimulus. The left panel shows saccade trajectories for the $\mathrm{RF}_{\text {correct }}^{\mathrm{T}}$ (when a target item fell in the $\mathrm{RF}$ and the monkey responded correctly) and $\mathrm{RF}_{\text {correct }}^{\mathrm{D}}$ (when a distractor fell in the RF and the monkey responded correctly) conditions in a representative session. The right panel shows the opposing trial types (black traces), when a target fell in the RF and a saccade was made away $\left(\mathrm{RF}_{\text {error }}^{\mathrm{T}}\right)$ as well as when a distractor fell in the RF and a saccade was made into the $\mathrm{RF}\left(\mathrm{RF}_{\mathrm{error}}^{\mathrm{D}}\right)$.
Across the recording sessions, both monkeys demonstrated significantly slower RTs on error trials than on correct trials (Fig. $1 B$; monkey $S: t(35)=-2.47, P<0.05$; monkey $Q: t(117)=-10.71, P<0.001)$, but the difference was larger for monkey $Q\left(M_{\text {Correct }}=318 \mathrm{~ms} ; M_{\text {Error }}=431\right.$ $\mathrm{ms})$ than for monkey $S\left(M_{\text {Correct }}=267 \mathrm{~ms} ; M_{\text {Error }}=279 \mathrm{~ms}\right)$. Error rates for monkey $Q$ were much lower (12\%) than for monkey $S$ ( $\left.35 \% ; U=779, n_{1}=118, n_{2}=36, P<0.001\right)$. This pattern of RTs and accuracy suggest that the two monkeys may have been operating with different speed-accuracy tradeoff criteria (Lohman 1989). Most importantly, both RT and error rate tended to increase with search set size (Fig. 1B). A long history of research implicates this "set size effect" as a signature of attention, as it is absent in conditions allowing preattentive selection of target stimuli (Treisman and Gelade 1980). A statistically significant increase in RT with set size was observed for monkey $Q$ [correct trials: $F(1,105)=902.38, P<$ 0.001; error trials: $F(1,104)=348.40, P<0.001]$ and for monkey $S$ [correct trials: $F(1,34)=177.27, P<0.001$; error trials: $F(1,34)=146.90, P<0.001]$. The same linear increase with set size was observed for error rate [monkey $Q: F(1,105)=$ 117.54, $P<0.001$; monkey $S: F(1,34)=155.26, P<0.001]$. We have previously documented the relationship between neural activity and the set size effect (Cohen et al. 2009b) and so will not discuss this further here.

\section{N2pc and FEF signals during correct and error trials}

Figure 2 shows the average m-N2pc simultaneously recorded with a single FEF neuron and associated LFP in a representative session (same session shown in Fig. 1A). On correct trials (black lines), all three signals selected the targets (thick lines) rather than distractors (thin lines) through characteristic modulation (Cohen et al. 2009a). The m-N2pc component exhibited a larger positivity for targets than for distractors in the RF, as previously shown (Cohen et al. 2009a; Woodman et al. 2007). Neurons expressed target selection by firing at a higher rate when target stimuli fell in the $\mathrm{RF}\left(\mathrm{RF}_{\text {correct }}^{\mathrm{T}}\right)$ relative to when distractors appeared there $\left(\mathrm{RF}_{\text {correct }}^{\mathrm{D}}\right)$, whereas LFPs demonstrated a more negative potential for target stimuli. To compare this pattern to that recorded on error trials, we measured the three types of signal (m-N2pc, spikes, and LFPs) when distractor items fell in the RF but an erroneous saccade was made toward it $\left(\mathrm{RF}_{\mathrm{error}}^{\mathrm{D}}\right)$ and trials in which a target stimulus fell in the RF but a saccade was made away $\left(\mathrm{RF}_{\mathrm{error}}^{\mathrm{T}}\right)$. Consistent with previous analyses of unit activity during visual search for color singletons or color-shape conjunctions (Thompson et al. 2005), neurons demonstrated reversed selectivity on error trials. That is, when an errant saccade was to be made into the RF, neurons responded as if the distractor was actually a target.

We now report two new observations. First, the reversed pattern of polarization was observed in the FEF LFP on error trials relative to correct trials. Second, when monkeys made search errors, the m-N2pc also treated the misidentified distractor as a target and the missed target as a distractor. That is, the selectivity is inverted for error trials relative to correct trials, and this inversion holds across all three kinds of signals. This has not been reported before in either the literature on human N2pc or on monkey neurophysiology. 

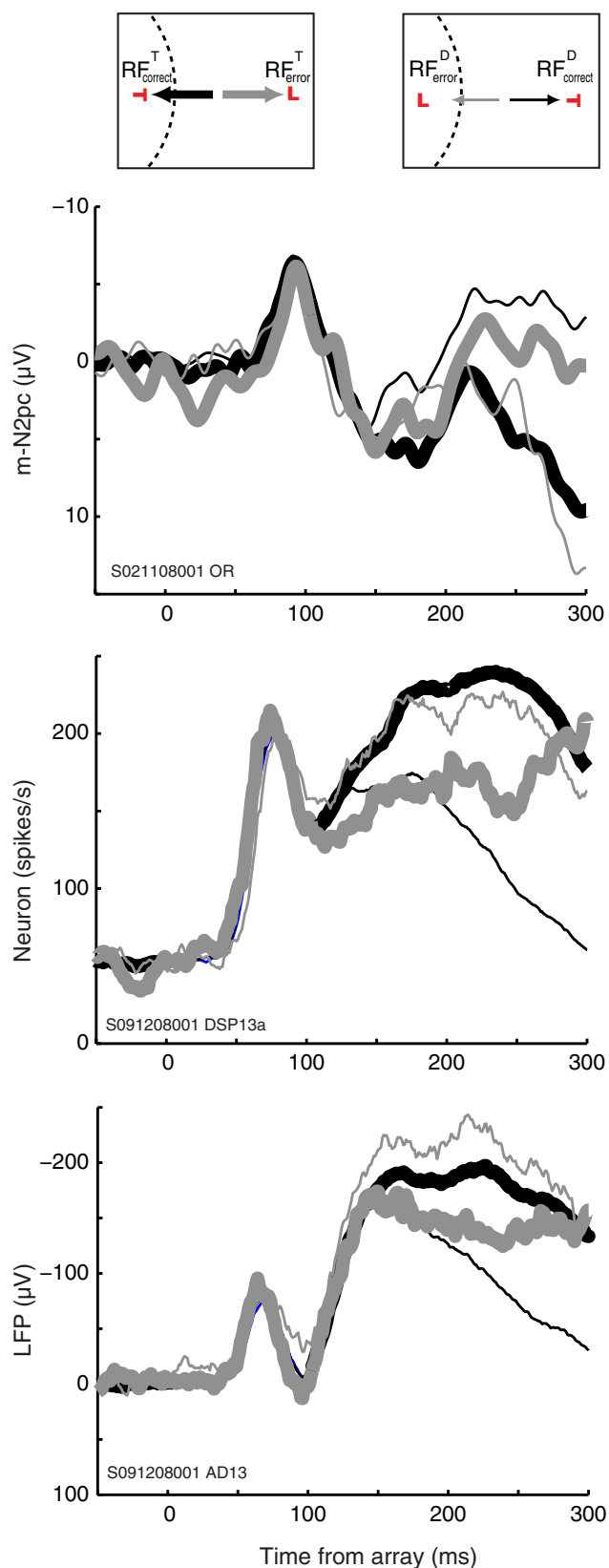

FIG. 2. Representative single-session averages for M-N2pc (top, electrode $\mathrm{OR}$ ), single neuron from frontal eye field (FEF, middle), and local field potential (LFP) from FEF (bottom).

As shown in Fig. 3, this pattern was replicated across the population in both monkeys. To evaluate these effects statistically, we compared the average firing rate or voltage within the period $150-250 \mathrm{~ms}$ after array appearance for $\mathrm{RF}^{\mathrm{T}}$ versus $\mathrm{RF}^{\mathrm{D}}$ trials. Statistics are combined across monkeys because the effects were indistinguishable. We carried out a mixed-model ANOVA with within-session factors of response type (correct versus incorrect) and stimulus array $\left(\mathrm{RF}^{\mathrm{T}}\right.$ vs. $\left.\mathrm{RF}^{\mathrm{D}}\right)$ and the between-session factor of signal (neuron vs. LFP vs. m-N2pc) because each session did not necessarily contribute to each of the signals. As illustrated in Fig. 3, the signals observed in $\mathrm{RF}^{\mathrm{T}}$ trials and $\mathrm{RF}^{\mathrm{D}}$ trials were significantly different, and the pattern of selectivity reversed between correct and error trials as evidenced by an interaction of response type $\mathrm{X}$ stimuli array for m-N2pc $[F(1,32)=72.2, P<0.001]$, neurons $[F(1,60)=$ 74.0, $P<0.001]$, and LFP $[F(1,76)=26.2, P<0.001]$. Surprisingly, single unit and LFP activity were sensitive to the presence of a target item in the RF even when an errant saccade was made away from that location. For neurons, $\mathrm{RF}_{\text {error }}^{\mathrm{T}}$ was significantly greater than $\mathrm{RF}_{\text {correct }}^{\mathrm{D}}, t(60)=-4.4, P<0.001$; for LFP, $\mathrm{RF}_{\text {error }}^{\mathrm{T}}$ was significantly less than $\mathrm{RF}_{\text {error }}^{\mathrm{D}}, t(76)=2.9$, $P<0.01$.

Figure 4 illustrates the quantitative pattern of selectivity on correct and error trials across the population. We subtracted the signal on $\mathrm{RF}^{\mathrm{D}}$ trials from that on $\mathrm{RF}^{\mathrm{T}}$ trials when the response was correct (abscissa) and when it was in error (ordinate) in the period 150-250 ms following array appearance. Each data point represents the direction of selectivity for one signal from one session. Signals that reversed polarity between correct and error trials will fall in the bottom-right quadrant (m-N2pc and single units) and the top-left quadrant (LFP). LFP data are opposite that of single units and $\mathrm{m}-\mathrm{N} 2 \mathrm{pc}$ because it demonstrates selection as a relative negativity rather than positivity. Signals that did not reverse between correct and error trials fall in the top-right or bottom-left quadrants. This illustrates the consistency of the reversal between correct and error trials across our sample. These findings show that tight linkage between the focus of selection by FEF activity and that of the m-N2pc is observed even when cognitive processing breaks down and the visual search task is performed incorrectly.

\section{Correlation of $N 2 p c$ and FEF LFP}

Previously we showed that the amplitude of LFP polarization in FEF is correlated trial-by-trial with the amplitude of the m-N2pc on correct trials (Cohen et al. 2009a). We determined whether this relationship is present even when visual search was incorrectly performed by computing the trial-by-trial correlation between the integral of the FEF LFP and the integral of the m-N2pc from 100 $\mathrm{ms}$ after the array appeared until the saccade was initiated. Similar to our previous report, LFP and $\mathrm{m}-\mathrm{N} 2 \mathrm{pc}$ voltages were correlated for $\mathrm{RF}_{\text {correct }}^{\mathrm{T}}$ trials (median $r=0.29, P<0.05$ ) and $\mathrm{RF}_{\text {correct }}^{\mathrm{D}}$ trials (median $r=0.33, P<0.05$ ). Interestingly, this correlation across brain areas and signal levels was also observed before errors, whether the error was made toward the $\mathrm{RF}\left(\mathrm{RF}_{\text {error }}^{\mathrm{D}}\right.$; median $r=$ $0.25, P<0.05)$ or away from it $\left(\mathrm{RF}_{\text {error }}^{\mathrm{T}}\right.$; median $r=0.30, P<$ $0.05)$.

\section{Catch trials}

We have established that each of the neurophysiological signals we recorded selected a distractor stimulus prior to an errant response. We next investigated whether a relationship existed between neural activity and the type of error made. For this, we turned to sessions that included catch trials when no target appeared. To earn reward, monkeys were required to withhold making any response for $\geq 750 \mathrm{~ms}$. We distinguished trials in which monkeys made no eye movements during the trial (correct catch) from trials in which the monkey made a saccade to a distractor before the $750 \mathrm{~ms}$ deadline (false alarms). We also analyzed trials in which monkeys maintained fixation through the $750 \mathrm{~ms}$ deadline (correct catch) but then made a saccade to a distractor item after receiving reinforcement (late catch response). Late catch responses were relatively common, amounting to $56 \%$ of all responses on catch trials (across the population, 18,557/ 

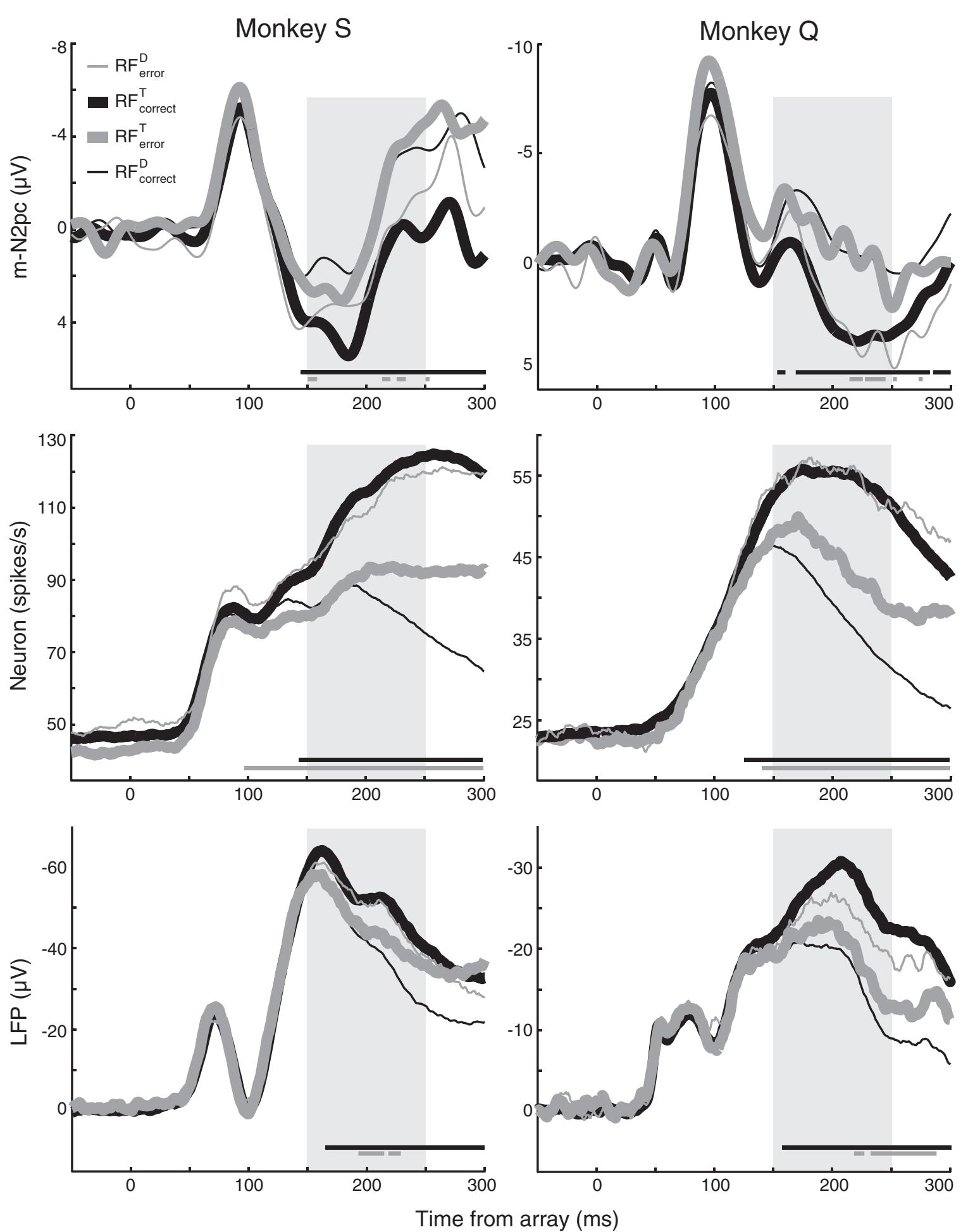

FIG. 3. Grand average m-N2pc (top), spike-density functions (middle), and LFP (bottom) for monkey $S$ (left) and monkey $Q$ (right). Traces are aligned to stimulus onset. Shaded region indicates the interval submitted to statistical test. Horizontal bars indicate times in which neural activation for $\mathrm{RF}^{\mathrm{T}}$ was significantly different from that for $\mathrm{RF}^{\mathrm{D}}$.

33,123). Because we did not train the monkeys extensively on catch trials, correct catch trials were less common $(17 \%, 5,694 /$ $33,123)$. Monkeys committed catch trial false alarms $\sim 27 \%$ of the time $(8872$ / 33123).

Among these various catch trial outcomes we identified neurons, LFPs, and ERPs according to the same criteria de- scribed previously. These yielded 17 ERPs, 18 neurons, and 13 LFPs. We then verified that saccade trajectories and landing points were similar between false alarms and late catch responses (Fig. 5A, see also Fig. 6B). Eye movements on correct catch trials were limited only to slow, drifting eye movements typically observed during the inter-trial interval. 


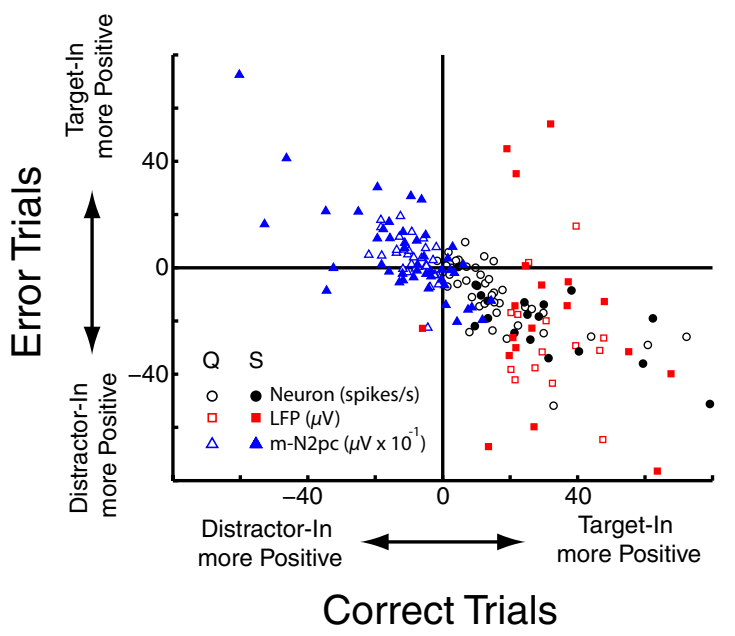

FIG. 4. Magnitude and direction of selectivity on correct (abscissa) and error (ordinate) trials across the population. Each point represents the average difference between $\mathrm{RF}^{\mathrm{T}}$ and $\mathrm{RF}^{\mathrm{D}}$ activity over the period $150-250 \mathrm{~ms}$ post stimulus onset for a given signal and session. Points falling in the top-right or bottom-left quadrants do not reverse polarity on correct and error trials. Those falling in the lower-right and upper left quadrants do reverse polarity (see text).

We contrasted neural activity preceding eye movements into the RF on catch trial false alarms and late catch responses as well as on target present trials when correct $\left(\mathrm{RF}_{\text {correct }}^{\mathrm{T}}\right)$ and errant $\left(\mathrm{RF}_{\text {correct }}^{\mathrm{D}}\right)$ saccades were produced into the receptive field. For single units in FEF (Fig. 5B), the activity on false alarm trials was not statistically different from that on $\mathrm{RF}_{\text {correct }}^{\mathrm{T}}$ trials $[t(16)=1.2, \mathrm{NS}]$. This finding replicates a previous report (Thompson et al. 2005). We now report an original observation; spike rate before late catch responses was significantly less than that on catch trial false alarm trials $[t(16)=$ $-4.20, P<0.001]$. The spike rate was, though, elevated relative to that measured when a saccade was correctly made away from the receptive field $\left(\mathrm{RF}_{\text {correct }}^{\mathrm{DT}}\right)[t(15)=4.99, P<$ $0.001]$. Activity on $\mathrm{RF}_{\text {correct }}^{\mathrm{D}}$ trials was not statistically distinguishable from that on correct catch trials $[t(16)=0.90, \mathrm{NS}]$. To summarize, when monkeys committed a false alarm, the FEF visual neurons responded the same as they did during correct trials. This mimics the pattern of activity observed when erroneous saccades were made to distractors when the target was present. However, when the monkey made a selfgenerated saccade to a distractor location after receiving reward, neural activity was significantly attenuated. This occurred despite the fact that saccade metrics were closely matched between the conditions.

We determined whether this attenuation persisted through the initiation of the saccade by limiting our analysis to response-aligned visuomovement neurons, which demonstrate a rapid increase in firing rate leading up to a saccadic response. Because each of these categories of saccades have effectively identical trajectories and landing points, one might expect that presaccadic activity is generated whether the saccade was correct, a false alarm, or a late catch response. Surprisingly, the firing rate of the neurons was at the baseline level immediately before late saccades after successful catch trials in contrast to the pronounced level of activity before any other kind of saccade into the RF (Fig. 5C). We verified that these neurons did in fact exhibit response-related activity during the memoryguided task. One representative neuron (Fig. 6A) clearly dem- onstrated delay period activity following array onset, and a later buildup of activity around the time of saccade. This neuron was sensitive to target stimuli in the bottom-left quadrant. Saccades directed to target stimuli in the cell's RF and to distractor stimuli during catch trials were quite similar (Fig. $6 B$ ). Most importantly, this neuron that responded before memory-guided saccades was virtually silent (perhaps even suppressed) before late catch saccades (Fig. 6C).

Consistent with the hypothesis that the FEF LFP is a manifestation of local cortical processing during visual search (Cohen et al. 2009a; Monosov et al. 2008), LFP polarization on catch trials was similar to what we observed in the firing rates of single neurons (Fig. 7). LFP polarization on false alarm trials was statistically indistinguishable from that on $\mathrm{RF}_{\text {correct }}^{\mathrm{T}}$ trials but was significantly more negative than that on $\mathrm{RF}_{\text {correct }}^{\mathrm{D}}$ trials $[t(12)=$
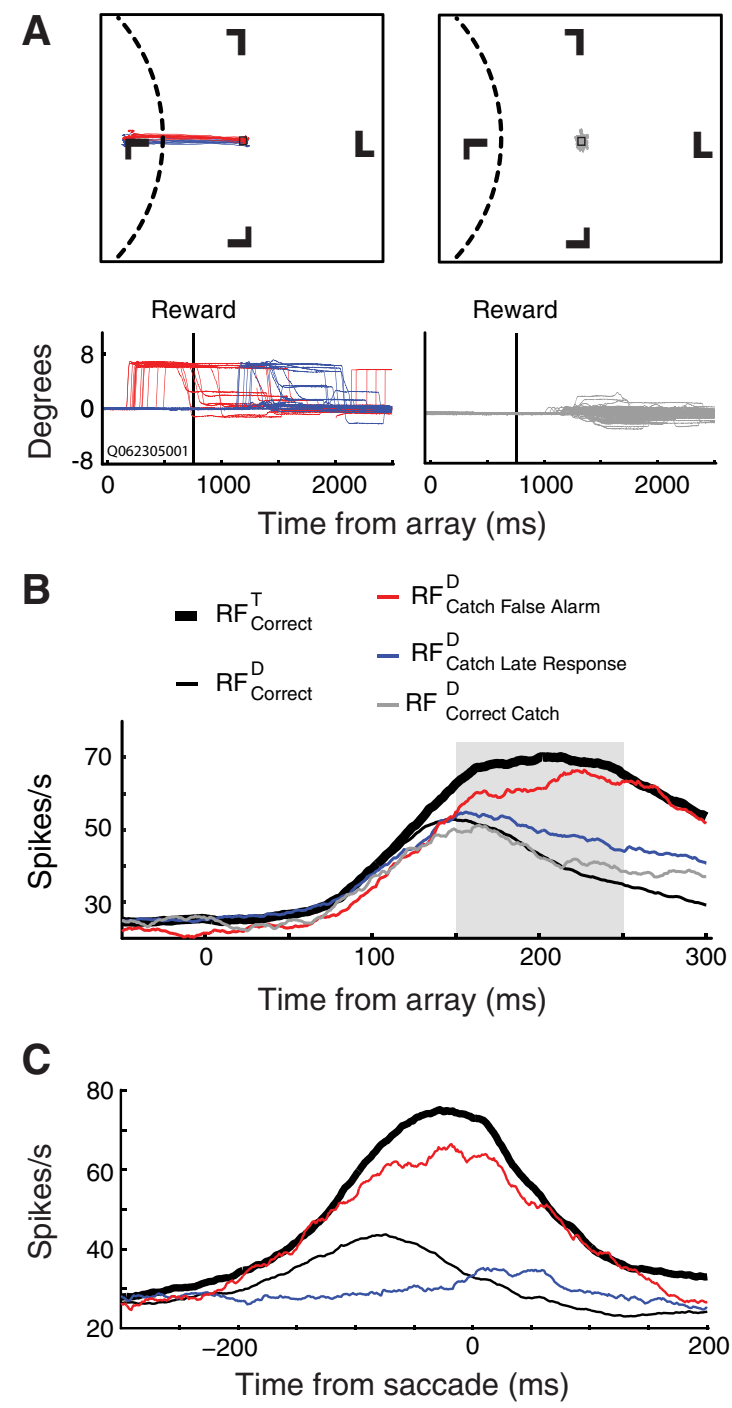

FIG. 5. A: saccade trajectories and $\mathrm{x}$-dimension eye traces (converted to degrees visual angle) for 1 representative session. Left: saccade trajectories and landing points during false alarms (red) and late catch responses (blue) are identical. Right: eye traces during correct catch trials, where no eye movement was detected. Small, nonzero voltages are slow drifting eye-movements typically observed during the inter-trial interval. $B$ : grand average spikedensity function on target-present and catch trials aligned to array onset. Shaded area indicates region submitted to statistical test. $C$ : grand average spike-density function on target-present and catch trials aligned to saccade initiation. 

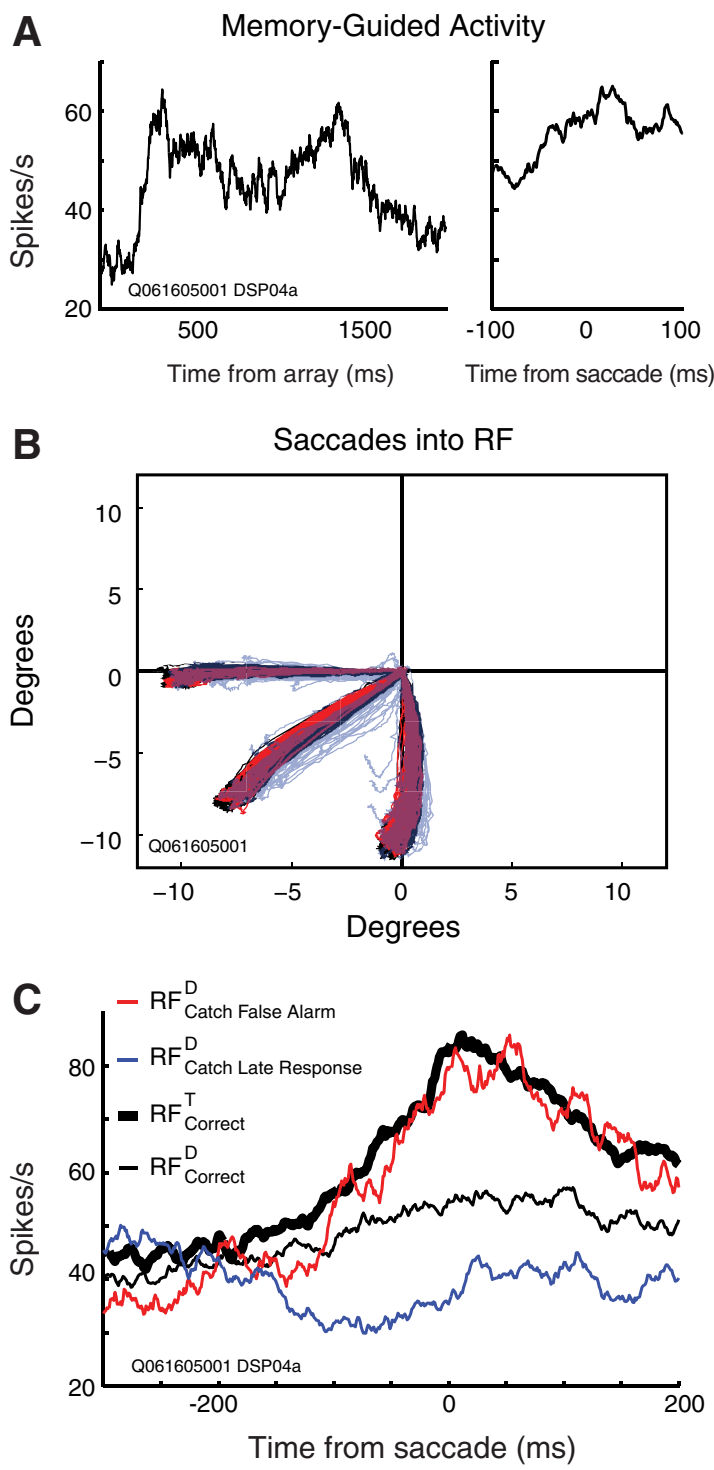

FIG. 6. A: memory-guided response profile for a typical visuomovement FEF neuron aligned to stimulus onset (left) and saccade onset (right). The neuron fired at elevated rates during the $1,000 \mathrm{~ms}$ memory interval before increasing again prior to movement. $B$ : saccade trajectories for $\mathrm{RF}_{\text {correct }}^{\mathrm{T}}$ trials (black), false alarms (red), and late catch responses (blue) into the RF of neuron in $A$. $C$ : response-aligned spike density functions for the same single neuron.

2.6, $P<0.05]$. The polarization on correct catch trials and late catch trial responses was not different.

A different pattern was evident in the $\mathrm{m}-\mathrm{N} 2 \mathrm{pc}$. The polarization in $\mathrm{RF}_{\text {correct }}^{\mathrm{T}}$, correct catch and late catch trials were statistically indistinguishable (Fig. 7). Meanwhile, significantly greater positivity was measured on false alarm trials relative to the voltage on $\mathrm{RF}_{\text {correct }}^{\mathrm{T}}$ trials $[t(7)=-4.07, P<0.01]$, and the polarization on $\mathrm{RF}_{\text {correct }}^{\mathrm{D}}$ trials was significantly different from that in all other conditions $[F(1,7)=44.62, P<0.001]$.

\section{I S C U S S I O N}

\section{N2pc and FEF signals during visual search errors}

The N2pc is generated by areas in parietal and occipitotemporal cortex (Hopf et al. 2000, 2004) that are reciprocally connected with FEF (Pouget et al. 2009; Schall et al. 1995b). Thus the N2pc could be considered a signature of the signals received by FEF. However, the projections of FEF to extrastriate visual cortex (Pouget et al. 2009) provide for the possibility that FEF influences visual processing (Moore and Armstrong 2003). In a recent study, we demonstrated that visually responsive neurons in macaque frontal eye field (FEF) could be a source of the feedback to that generates the m-N2pc measured over posterior visual areas (Cohen et al. 2009a). Specifically, we found that the spiking activity and LFP in FEF selected visual search targets significantly earlier than did the $\mathrm{m}-\mathrm{N} 2 \mathrm{pc}$. Thus the timing and form of FEF activity when visual search is correctly performed are consistent with the hypothesis that signals from FEF drive the extrastriate attentional selection mechanism manifest as the m-N2pc. This hypothesis is bolstered by the recent observation that the enhancement of neural activity due to attention occurs earlier in FEF neurons than in V4 neurons (Gregoriou et al. 2009; Ogawa and Komatsu 2006) as well as the demonstration that subthreshold microstimulation of FEF produces an enhancement of V4 neuron activity (Armstrong et al. 2006; Moore and Armstrong 2003).

Although the direction of influence cannot be determined by the present data, the results do strengthen the link between target selection in FEF and the covert orienting of attention. Previous studies reveal that single-unit responses in FEF and superior colliculus incorrectly select distractor stimuli prior to an errant response (Shen and Paré 2007; Thompson et al. 2005). That is, single units fire at a higher rate when distractor stimuli fall in the receptive field (RF) prior to an incorrect saccade to that distractor compared with when a correct response is made to a target outside the RF. We found that the macaque homologue of the human N2pc component mirrors activity in FEF even before errors. To our knowledge, such a pattern has never been reported in the human or non-human primate literature.

Alternatively, it was entirely possible that the m-N2pc would be absent during errant responses. Because the m-N2pc
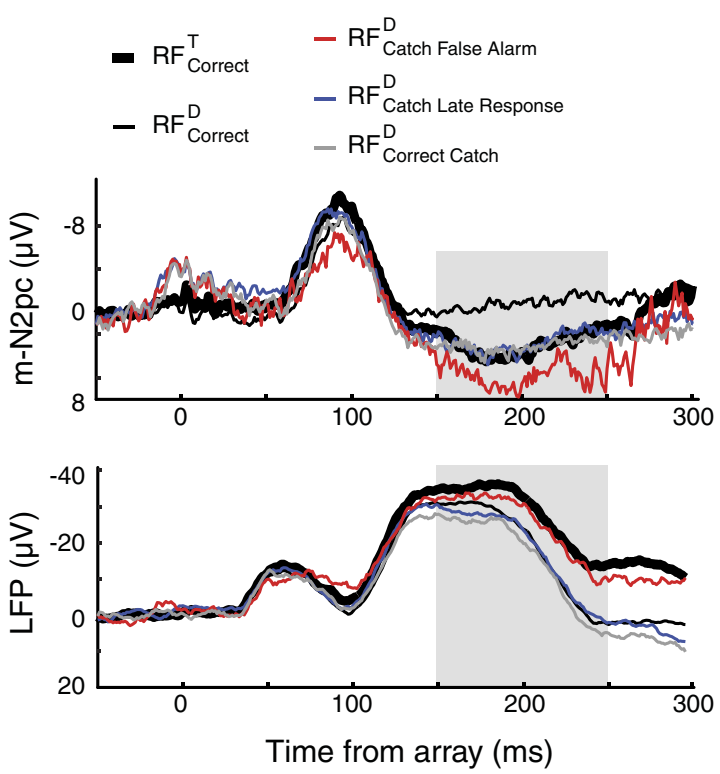

FIG. 7. Grand average m-N2pc (top) and LFP (bottom) on correct targetpresent and catch trials. Shaded area indicates region submitted to statistical test. 
is a lateralized component in humans and non-human primates, it can only emerge under specific circumstances, and previous research has documented a number of task conditions that fail to elicit it (Luck and Hillyard 1994). If errors during visual search entailed a nonsystematic orienting of attention to items across the visual field, the $\mathrm{m}-\mathrm{N} 2 \mathrm{pc}$ would not develop on error trials though present before correct responses. Similarly, if FEF activity did not reflect the orienting of attention, or if the error occurred during response mapping, one might expect to see accurate orienting of attention reflected in the m-N2pc despite the patterns exhibited by FEF. Indeed when response mapping errors are more probable, FEF neurons correctly identify target stimuli both when report is correct and in error (Trageser et al. 2008). The present data make clear that errors during this visual search task stemmed from a systematic misorienting of attention and further support the identification of target selection in FEF with attention allocation.

Our analysis of the FEF LFPs provides further support for the relationship between the m-N2pc and FEF activity. First, like single unit responses, the FEF LFP incorrectly selected distractor stimuli prior to an error. This is perhaps not surprising as LFPs are thought to be a result of afferent postsynaptic potentials (Katzner et al. 2009; Mitzdorf 1985) and are related to local spiking (Fox and O'Brien 1965). Second, the amplitude of FEF activity was correlated with m-N2pc amplitude on a trial-by-trial basis. While this can neither establish the direction of influence nor discount common input as a potential mediator, it is clear that the cortical areas responsible for the $\mathrm{m}-\mathrm{N} 2 \mathrm{pc}$ are related to activity local to FEF. Cortical inactivation studies will be useful in exploring these possibilities. For instance, the viewpoint that FEF drives the attention-related effects observed in extrastriate cortex predicts the absence of the $\mathrm{m}-\mathrm{N} 2 \mathrm{pc}$ following FEF inactivation.

\section{Contingency of FEF signals}

This work demonstrates a new dissociation in neural activity related to the type of error produced. In FEF (single units and LFPs), catch trial false alarm saccades to a distractor in the receptive field were associated with activity closely resembling that recorded on trials when the target appeared in a neurons' receptive field $\left(\mathrm{RF}_{\text {correct }}^{\mathrm{T}}\right)$. This is intuitive, as both trial types involve saccades into the same RF. However, when monkeys, after receiving reward for a successful catch trial, made an accurate but late self-initiated, visually guided saccade to a distractor located in the RF, neural activity was significantly reduced. Unlike subcortical structures such as central thalamus (Schlag-Rey and Schlag 1984), cortical areas associated with eye movements (e.g., FEF, supplementary eye fields) exhibit little modulation during spontaneous saccades in the dark (Bruce and Goldberg 1985; Schall 1991). This is the first demonstration of a lack of FEF neural activity during seemingly purposive saccades. Surprisingly, visuomovement neuron activity aligned to saccade onset also demonstrated this pattern. In other words, neurons that are normally very active before a task-related saccade were silent when a saccade of the same direction was made after receiving reinforcement. It is possible that FEF may have been preparing a guess, and one signature of this may be the slight elevation of single unit activity prior to late catch responses (Fig. 5B). Many classic models of decision formation assume that guesses are either preselected or evolve during a trial (Meyer et al. 1988; Ollman and Billington 1972) to be enacted according to some internal deadline corresponding to the length of time allowed for stimulus processing. Such strategies become useful when trials are time-limited as in the present task. Probabilistically, monkeys stand to receive reinforcement at a higher rate by making a random guess than by withholding a saccade altogether. Because a guess by definition would not depend on the behavioral significance of any item, and because FEF neurons are sensitive to context, it stands to reason that activity will be attenuated. Alternatively, this may be a situation that dissociates attention and reward-constructs that are often necessarily confounded (Maunsell 2004) because the attenuation in neural activity was observed after reward delivery. However, as we cannot be sure of the nature of attentional orienting preceding such responses, any further conclusions would be speculative. In either case, it is interesting that FEF unit activity is nearly absent during the same eye movements that otherwise produce strong responses. These findings add to the evidence that the visual activity in FEF is not directly related to saccade preparation or planning (Hanes et al. 1998; Juan et al. 2004; Monosov et al. 2008; Murthy et al. 2009; Thompson et al. 1996).

The current work supports the role of FEF in the production of covert attention and further establishes a link between FEF and the cortical drivers of the m-N2pc measure of selective attention employed in both monkeys and humans. Further, these data make clear that errors during this visual search task were systematic mis-deployments of attention rather than a breakdown in attentional mechanisms per se. That the m-N2pc arises at all before behavioral errors reflects a consistency of perceptual processing and attentional selection even when that processing is flawed.

\section{A C K N O W LED G M E N T S}

We thank K. Ferguson and S. Denduluri for help with data analysis.

\section{G R A N T S}

This work was supported by National Institutes of Health Grants F32-EY019851 to R. P. Heitz, F32-EY-015043 to G. F. Woodman, and T32-MH064913, T32-EY-007135, R01-EY-08890, P30-EY-08126, P30-HD-015052 and Robin and Richard Patton through the E. Bronson Ingram Chair in Neuroscience.

\section{I S C L O S URES}

No conflicts of interest, financial or otherwise, are declared by the author(s).

\section{R E F ER E N C E S}

Armstrong KM, Chang MH, Moore T. Selection and maintenance of spatial information by frontal eye field neurons. J Neurosci 29: 15621-15629, 2009.

Armstrong KM, Fitzgerald JK, Moore T. Changes in visual receptive fields with microstimulation of frontal cortex. Neuron 50: 791-798, 2006.

Britten KH, Shadlen MN, Newsome WT, Movshon JA. The analysis of visual motion: a comparison of neuronal and psychophysical performance. J Neurosci 12: 4745-4765, 1992.

Bruce CJ, Goldberg ME. Primate frontal eye fields. I. Single neurons discharging before saccades. J Neurophysiol 53: 603-635, 1985.

Cohen JY, Heitz RP, Schall JD, Woodman GF. On the origin of eventrelated potentials indexing covert attentional selection during visual search. J Neurophysiol 102: 2375-2386, 2009a. 
Cohen JY, Heitz RP, Woodman GF, Schall JD. Neural basis of the set-size effect in frontal eye field: timing of attention during visual search. $J$ Neurophysiol 101: 1699-1704, 2009b.

Fox SS, O'Brien JH. Duplication of evoked potential waveform by curve of probability of firing of a single cell. Science 147: 888-890, 1965.

Gregoriou GG, Gotts SJ, Zhou H, Desimone R. High-frequency, long-range coupling between prefrontal and visual cortex during attention. Science 324: 1207-1210, 2009.

Hanes DP, Patterson WF, Schall JD. Role of frontal eye fields in countermanding saccades: visual, movement, and fixation activity. $J$ Neurophysiol 79: 817-834, 1998.

Hanes DP, Schall JD. Neural control of voluntary movement initiation. Science 274: 427-430, 1996.

Hikosaka O, Wurtz RH. Visual and oculomotor functions of monkey substantia nigra pars reticulata. IV. Relation of substantia nigra to superior colliculus. J Neurophysiol 49: 1285-1301, 1983.

Hopf JM, Boelmans K, Schoenfeld MA, Luck SJ, Heinze H-J. Attention to features precedes attention to locations in visual search: evidence from electromagnetic brain responses in humans. J Neurosci 24: 1822-1832, 2004.

Hopf JM, Luck SJ, Girelli M, Hagner T, Mangun GR, Scheich H, Heinze HJ. Neural sources of focused attention in visual search. Cereb Cortex 10: 1233-1241, 2000.

Jasper HH. The ten twenty electrode system of the international federation. Electroencephalogr Clin Neurophysiol 10: 371-375, 1958.

Juan CH, Shorter-Jacobi SM, Schall JD. Dissociation of spatial attention and saccade preparation. Proc Natl Acad Sci USA 101: 15541-15544, 2004.

Katzner S, Nauhaus I, Benucci A, Bonin V, Ringach DL, Carandini M. Local origin of field potentials in visual cortex. Neuron 61: 35-41, 2009.

Kodaka Y, Mikami A, Kubota K. Neuronal activity in the frontal eye field of the monkey is modulated while attention is focused on to a stimulus in the peripheral visual field, irrespective of eye movement. Neurosci Res 28 : 291-298, 1997.

Lohman DF. Individual differences in errors and latencies on cognitive tasks. Learning Individual Differences 1: 179-202, 1989.

Luck SJ, Fan S, Hillyard SA. Attention-related modulation of sensoryevoked brain activity in a visual search task. J Cogn Neurosci 5: 188-195, 1993.

Luck SJ, Hillyard SA. Spatial filtering during visual search: evidence from human electrophysiology. J Exp Psychol Hum Percept Perform 20: 1000 1014, 1994.

Maunsell JHR. Neuronal representations of cognitive state: reward or attention? Trends Cogn Sci 8: 261-265, 2004.

Meyer DE, Irwin DE, Osman AM, Kounios J. The dynamics of cognition and action: mental processes inferred from speed-accuracy decomposition. Psychol Rev 95: 183-237, 1988.

Mitzdorf U. Current source-density method and application in cat cerebral cortex: investigation of evoked potentials and EEG phenomena. Physiol Rev 65: 37-100, 1985.

Monosov IE, Trageser JC, Thompson KG. Measurements of simultaneously recorded spiking activity and local field potentials suggest that spatial selection emerges in the frontal eye field. Neuron 57: 614-625, 2008.

Moore T, Armstrong KM. Selective gating of visual signals by microstimulation of frontal cortex. Nature 421: 370-373, 2003

Murthy A, Ray S, Shorter SM, Schall JD, Thompson KG. Neural control of visual search by frontal eye field: effects of unexpected target displacement on visual selection and saccade preparation. J Neurophysiol 101: 2485-2506, 2009
Nelson MJ, Pouget P, Nilsen EA, Patten CD, Schall JD. Review of signal distortion through metal microelectrode recording circuits and filters. $J$ Neurosci Methods 169: 141-157, 2008.

Ogawa T, Komatsu H. Neuronal dynamics of bottom-up and top-down processes in area V4 of macaque monkeys performing a visual search. Exp Brain Res 173: 1-13, 2006.

Ollman RT, Billington MJ. The deadline model for simple reaction times. Cogn Psychol 3: 311-336, 1972.

Pouget P, Stepniewska I, Crowder EA, Leslie MW, Emeric EE, Nelson MJ, Schall JD. Visual and motor connectivity and the distribution of calcium-binding proteins in macaque frontal eye field: implications for saccade target selection. Front Neuroanat 3: 2, 2009.

Purcell, BA, Heitz, RP, Cohen, JY, Schall, JD, Logan, GD, Palmeri, TJ. Neurally constrained modeling of perceptual decision making. Psychol Rev In press.

Rabbitt PM. Errors and error correction in choice-response tasks. $J$ Exp Psychol 71: 264-272, 1966.

Ratcliff R, Rouder J. Modeling response times for two-choice decisions. Psychol Sci 9: 347-356, 1998.

Ray S, Schall JD, Murthy A. Programming of double-step saccade sequences: modulation by cognitive control. Vision Res 44: 2707-2718, 2004.

Sato TR, Schall JD. Effects of stimulus-response compatibility on neural selection in frontal eye field. Neuron 38: 637-648, 2003.

Schall JD. Neuronal activity related to visually guided saccades in the frontal eye fields of rhesus monkeys: comparison with supplementary eye fields. $J$ Neurophysiol 66: 559-579, 1991.

Schall JD, Hanes DP, Thompson KG, King DJ. Saccade target selection in frontal eye field of macaque. I. Visual and premovement activation. $J$ Neurosci 15: 6905-6918, 1995a.

Schall JD, Morel A, King DJ, Bullier J. Topography of visual cortex connections with frontal eye field in macaque: convergence and segregation of processing streams. J Neurosci 15: 4464-4487, 1995 b.

Schlag-Rey M, Schlag J. Visuomotor functions of central thalamus in monkey. I. Unit activity related to spontaneous eye movements. $J$ Neurophysiol 51: 1149-1174, 1984

Shen K, Paré M. Neuronal activity in superior colliculus signals both stimulus identity and saccade goals during visual conjunction search. $J$ Vision 7: 15.11-13, 2007.

Thompson KG, Bichot NP, Sato TR. Frontal eye field activity before visual search errors reveals the integration of bottom-up and top-down salience. $J$ Neurophysiol 93: 337-351, 2005.

Thompson KG, Hanes DP, Bichot NP, Schall JD. Perceptual and motor processing stages identified in the activity of macaque frontal eye field neurons during visual search. J Neurophysiol 76: 4040-4055, 1996.

Trageser JC, Monosov IE, Zhou Y, Thompson KG. A perceptual representation in the frontal eye field during covert visual search that is more reliable than the behavioral report. Eur J Neurosci 28: 2542-2549, 2008.

Treisman AM, Gelade G. A feature-integration theory of attention. Cogn Psychol 12: 97-136, 1980.

Woodman GF, Kang MS, Rossi AF, Schall JD. Nonhuman primate eventrelated potentials indexing covert shifts of attention. Proc Natl Acad Sci USA 104: 15111-15116, 2007.

Woodman GF, Luck SJ. Electrophysiological measurement of rapid shifts of attention during visual search. Nature 400: 867-869, 1999.

Woodman GF, Luck SJ. Dissociations among attention, perception, and awareness during object-substitution masking. Psychol Sci 14: 605-611, 2003. 